\title{
Status of cardiovascular PET radiation exposure and strategies for reduction: An Information Statement from the Cardiovascular PET Task Force
}

\author{
James A. Case, PhD, a Robert A. deKemp, PhD, , Piotr J. Slomka, PhD, ${ }^{c}$ \\ Mark F. Smith, PhD, ${ }^{d}$ Gary V. Heller, MD, PhD, ${ }^{e}$ and Manuel D. Cerqueira, $M^{f}$ \\ a Cardiovascular Imaging Technologies, L.L.C, Kansas City, MO \\ b University of Ottawa Heart Institute, Ottawa, Canada \\ c Cedars-Sinai Medical Center, Los Angeles, CA \\ d University of Maryland, Baltimore, MD \\ e Gagnon Cardiovascular Institute, Morristown Medical Center, Morristown, NJ \\ f Cleveland Clinic Foundation, Cleveland, $\mathrm{OH}$
}

Received Mar 23, 2017; accepted Mar 23, 2017

doi: 10.1007/s12350-017-0897-9

Cardiovascular positron emission tomography (PET) imaging provides high-quality visual and quantitative myocardial perfusion and function images. In addition, cardiovascular PET can assess myocardial viability, myocardial inflammatory disorders such as cardiac sarcoid, and infections of implanted devices including pacemakers, ventricular assist devices, and prosthetic heart valves. As with all nuclear cardiology procedures, the benefits need to be considered in relation to the risks of exposure to radiation. When performed properly, these assessments can be obtained while simultaneously minimizing radiation exposure. The purpose of this information statement is to present current concepts to minimize patient and staff radiation exposure while ensuring high image quality.

Key Words: PET imaging $\cdot$ radiation protection $\cdot$ myocardial perfusion imaging: PET

\section{Cardiovascular PET Task Force}

Brian G. Abbott, MD

Timothy M. Bateman, MD

Rob S.B. Beanlands, MD

Manuel D. Cerqueira, MD

E. Gordon DePuey, MD

Gary V. Heller, MD, PhD

Justin B. Lundbye, MD

Patrick White

David C. Wolinsky, MD

Reprint requests: James A. Case, $\mathrm{PhD}$, Cardiovascular Imaging

Technologies, L.L.C, Kansas City, MO; jcase@cvit.com

J Nucl Cardiol 2017;24:1427-39.

$1071-3581 / \$ 34.00$

Copyright (C) 2017 American Society of Nuclear Cardiology.

\section{INTRODUCTION}

The use of radionuclide imaging has improved the diagnosis and management of patients with known or suspected coronary artery disease and heart failure. The benefits of single-photon emission computed tomography (SPECT) and positron emission tomography (PET) are widely recognized and clinical studies are performed worldwide. The International Atomic Energy Agency, while recognizing the risk of ionizing radiation, reaffirmed the importance of imaging, " .... it is hoped that its (radiological imaging) use in medicine will increase, as the benefits for patients are enormous, far exceeding the risks." ${ }^{1}$ It is essential that these benefits are achieved using the procedures that minimize the patient's risk from radiation. The United States Food and Drug Administration and the American Society of Nuclear Cardiology (ASNC) recognize that image quality and reducing radiation exposure are not mutually exclusive 
goals; "Lowering the radiation while maintaining or improving image quality should be considered an improvement in the quality of care', 2,3

For both SPECT and PET, a step-by-step approach should be followed in developing flexible protocols that maximize patient benefit, while minimizing risk. Facilities should not sacrifice diagnostic accuracy for the sake of reducing radiation. Recognized steps include the following:

(1) Establish a clear definition of the clinical question and the appropriateness of the nuclear study based on appropriate use criteria.

(2) Consider individual risks for exposure to ionizing radiation: age, risk profile, and gender, especially women of child bearing potential.

(3) Optimize radiation dose to the patient; consider patient body habitus, number of images acquired, and machine settings.

(4) Consider all sources of radiation exposure, including transmission scans.

(5) Consider radiation exposure to the staff and the public.

Extensive literature on techniques to reduce radiation exposure in the performance of SPECT studies has been reported. ${ }^{4}$ However, less information is available on exposure from current radiopharmaceutical dosages and strategies for dose reduction with cardiovascular PET. This paper will focus on current PET radiation exposure to patients and how to reduce exposure while maintaining diagnostic accuracy. Definitions of radiation measures and their units are given in Appendix 1.

\section{PET RADIATION SOURCES}

\section{PET Radiotracers}

The most commonly used cardiac PET tracers for assessment of qualitative myocardial perfusion and quantitative measurement of absolute myocardial blood flow $(\mathrm{MBF})$ are rubidium-82 chloride $\left({ }^{82} \mathrm{Rb}\right)$ and nitrogen-13 $\left({ }^{13} \mathrm{~N}\right)$ ammonia. Fluorine-18 $\left({ }^{18} \mathrm{~F}\right)$ fluorodeoxyglucose $\left({ }^{18} \mathrm{~F}\right.$-FDG) is used to identify hibernating myocardium, to diagnose cardiac sarcoid, and to identify sites of myocardial device infection and other inflammatory conditions.

The injected tracer activity (sometimes referred to as injected dose) is expressed in units of millicuries $(\mathrm{mCi})$ or megabecquerels $(\mathrm{MBq})$. However, conversion to effective dose, in units of millisieverts ( $\mathrm{mSv}$ ) using a tracer-specific effective dose coefficient (EDC), makes it possible to estimate the effective radiation dose and relate it to other medical procedures such as $\mathrm{x}$-ray CT and diagnostic angiography. A comparison of patient radiation dose for various SPECT and PET protocols using current International Commission on Radiological Protection (ICRP) conversions is shown in Table $1.5,6$

Table 1. Common PET and SPECT protocols and radiation effective doses (E) $5,6,10$

\begin{tabular}{|c|c|c|c|c|}
\hline Study protocol & Isotope & Modality & $\begin{array}{l}\text { Activity } \\
(\mathrm{mCi})^{a}\end{array}$ & $\mathbf{E}(\mathbf{m} \mathbf{b} \mathbf{v})$ \\
\hline \multirow[t]{2}{*}{ Rest + stress perfusion } & \multirow[t]{2}{*}{${ }^{82} \mathrm{Rb}$-chloride } & 3D PET & $25+25$ & 2 \\
\hline & & 2D PET & $50+50$ & 4 \\
\hline \multirow[t]{2}{*}{ Rest + stress perfusion } & \multirow[t]{2}{*}{${ }^{13} \mathrm{~N}$-ammonia } & 3D PET & $10+10$ & 2 \\
\hline & & 2D PET & $20+20$ & 4 \\
\hline \multirow{2}{*}{$\begin{array}{l}\text { Rest viability, sarcoid, or inflammation } \\
\text { (+ perfusion) }\end{array}$} & \multirow[t]{2}{*}{${ }^{18} \mathrm{~F}-\mathrm{FDG}\left(+{ }^{13} \mathrm{NH}_{3}\right.$ or $\left.{ }^{82} \mathrm{Rb}\right)$} & 3D PET & $5(+10$ or 25$)$ & $3.5(+1)$ \\
\hline & & 2D PET & $10(+20$ or 50$)$ & $7(+2)$ \\
\hline Stress-only perfusion (ultra-low-dose) & ${ }^{99 m}$ Tc-sestamibi & CZT-SPECT & 3.5 & 1 \\
\hline Stress-only perfusion (full-dose) & ${ }^{99 m}$ Tc-sestamibi & GC-SPECT & 30 & 10 \\
\hline \multirow[t]{2}{*}{ Rest + stress perfusion one-day (half-dose) } & ${ }^{99 \mathrm{~m}}$ Tc-sestamibi & GC-SPECT & $5+15$ & 6.4 \\
\hline & ${ }^{99 \mathrm{~m}}$ Tc-tetrofosmin & & & 5.6 \\
\hline \multirow{2}{*}{ Rest + stress perfusion one-day (full-dose) } & ${ }^{99 \mathrm{~m}}$ Tc-sestamibi & GC-SPECT & $10+30$ & 13 \\
\hline & ${ }^{99 \mathrm{~m}}$ Tc-tetrofosmin & & & 11 \\
\hline
\end{tabular}

CZT cadmium zinc telluride, GC gamma camera

Radiation effective dose $(\mathrm{E})$ : activity $(\mathrm{mCi}) \times 37(\mathrm{MBq} / \mathrm{mCi}) \times$ effective dose constant $(\mathrm{mSv} / \mathrm{MBq})=\mathrm{effective} \mathrm{dose}(\mathrm{mSv})$

Effective dose constants: ${ }^{82} \mathrm{Rb}$-chloride $=1.1 \times 10^{-3} \mathrm{mSv} / \mathrm{MBq} ;{ }^{13} \mathrm{~N}$-ammonia $=2.7 \times 10^{-3} \mathrm{mSv} / \mathrm{MBq} ;{ }^{18} \mathrm{~F}-\mathrm{FDG}=1.9 \times 10^{-2}$ $\mathrm{mSv} / \mathrm{MBq} ;{ }^{99 \mathrm{~m}} \mathrm{Tc}$-sestamibi $=8.0 \times 10^{-3} \mathrm{mSv} / \mathrm{MBq}$ (stress), $7.9 \times 10^{-3} \mathrm{mSv} / \mathrm{MBq}$ (rest); ${ }^{99 \mathrm{~m}} \mathrm{Tc}$-tetrofosmin $=8.0 \times 10^{-3} \mathrm{mSv} /$ $\mathrm{MBq}$ (stress), $6.3 \times 10^{-3} \mathrm{mSv} / \mathrm{MBq}$ (rest)

aDoses listed are typical for cardiac PET and can vary for specific instrumentation and application. For specific dose recommendation, refer to the appropriate imaging guidelines ${ }^{15}$ and manufacturer prescribing information

${ }^{b}$ Effective doses listed are based on the ICRP Publication $128^{10}$ and may differ from prescribing information 
Rubidium-82. ${ }^{82} \mathrm{Rb}$ is a potassium analog with a half-life of about 75 seconds and is actively transported across the cell membrane by the sodium-potassium ATPase transport system. Because the half-life of ${ }^{82} \mathrm{Rb}$ is short, it is ideal for sequential rest and pharmacologic stress studies.

${ }^{82} \mathrm{Rb}$ is produced from a strontium- $82\left({ }^{82} \mathrm{Sr}\right) /{ }^{82} \mathrm{Rb}$ generator and administered using an infusion pump system. The eluate from the ${ }^{82} \mathrm{Sr} /{ }^{82} \mathrm{Rb}$ generator must be monitored daily before first patient use to ensure that any contamination with long-lived ${ }^{82} \mathrm{Sr}$ and strontium- $85\left({ }^{85} \mathrm{Sr}\right)$ is below the United States Pharmacopeia (USP) limits.

The monitoring for ${ }^{82} \mathrm{Sr}$ and ${ }^{85} \mathrm{Sr}$ breakthrough is one of the most important quality assurance steps. As the ${ }^{82} \mathrm{Rb}$ generator is used, the amount of these contaminants in the eluate increases. For the Cardiogen ${ }^{\circledR}-82$ ${ }^{82} \mathrm{Rb}$ generator, the manufacturer requires increased ${ }^{82} \mathrm{Sr}$ and ${ }^{85} \mathrm{Sr}$ monitoring after $14 \mathrm{~L}$ of eluate volume is drawn from the system and discontinuation of use of the generator after $17 \mathrm{~L}^{7}{ }^{7}$ For the RUBY-FILL ${ }^{\circledR}$ system, the manufacturer requires increased ${ }^{82} \mathrm{Sr}$ and ${ }^{85} \mathrm{Sr}$ monitoring after $20 \mathrm{~L}$ of eluate volume is drawn from the system and discontinuation of use of the generator after $30 \mathrm{~L} .{ }^{8}$

Radiation dosimetry estimates acquired on modern PET/CT imaging equipment suggest an average patient dose of 2-4 mSv for a ${ }^{82} \mathrm{Rb}$ rest/stress protocol depending on the injected activities and imaging protocol used (see Table 1). These estimates for ${ }^{82} \mathrm{Rb}$ represent the current expert consensus and product labeling on ${ }^{82} \mathrm{Rb}$ dosimetry, though some estimates of radiation dose may be lower. ${ }^{9-11}$

${ }^{13} \mathrm{~N}$ ammonia. ${ }^{13} \mathrm{~N}$ ammonia is synthesized by proton bombardment of water in a cyclotron to yield ${ }^{13} \mathrm{~N}$ nitrates, followed by reduction reactions to produce ${ }^{13} \mathrm{~N}$ ammonia. ${ }^{12}{ }^{13} \mathrm{~N}$ ammonia is taken up by the myocytes and is trapped as ${ }^{13} \mathrm{~N}$ glutamine via the glutamic acidglutamine pathway. ${ }^{12,13}{ }^{13} \mathrm{~N}$ ammonia has demonstrated excellent first-pass extraction and reproducible flow characteristics, ${ }^{13}$ and can be administered for sequential rest and stress studies due to the short 10 minute half-life of ${ }^{13} \mathrm{~N}$. Generally, ${ }^{13} \mathrm{~N}$ ammonia is administered as 10 $20 \mathrm{mCi}$ (370-740 MBq) for each rest and stress injection.

${ }^{18}$ F-Fluorodeoxyglucose. Myocardial viability, inflammation, and infection can be assessed with ${ }^{18} \mathrm{~F}$ fluorodeoxyglucose $\left({ }^{18} \mathrm{~F}\right.$ FDG $) .{ }^{18} \mathrm{~F}$ is cyclotron-produced by proton irradiation of ${ }^{18} \mathrm{O}$ water and has a 110 minute half-life. Deoxyglucose is then labeled with ${ }^{18} \mathrm{~F}$ to yield ${ }^{18} \mathrm{~F}$ FDG, an analog of glucose. ${ }^{14}$ Standardized patient preparation protocols are employed to ensure either adequate ${ }^{18} \mathrm{~F}$ FDG uptake in the case of viability studies, or to inhibit normal myocardial uptake in the case of cardiac inflammation studies. ${ }^{15,16}$

To evaluate myocardial viability, infection, or inflammation using ${ }^{18} \mathrm{~F}$ FDG, a comparator rest or stress perfusion study is required, and should be part of the radiation exposure calculation for the study. ${ }^{82} \mathrm{Rb}$ and

${ }^{13} \mathrm{~N}$ ammonia are the best perfusion comparators in terms of radiation dose and imaging comparability to ${ }^{18} \mathrm{~F}$ FDG, but if a SPECT study is available, it may be used, preferably with attenuation correction applied. Injected activities of $5-15 \mathrm{mCi}(185-555 \mathrm{MBq}){ }^{18} \mathrm{~F}$ FDG are acceptable and $10 \mathrm{mCi}(370 \mathrm{MBq})$ is most commonly used for 2D PET imaging. The estimated radiation dose for a $10 \mathrm{mCi}(370 \mathrm{MBq})$ injection of ${ }^{18} \mathrm{~F} \mathrm{FDG}$ is $7 \mathrm{mSv}{ }^{5}$

\section{Radiation from Transmission Sources}

All myocardial PET protocols utilize a patientspecific transmission scan for correction of soft tissue attenuation. Early PET systems relied on ring or rotating isotope line or point sources, but 3D PET/CT hybrid systems are most commonly used today with $\mathrm{x}$-ray transmission. Depending on the protocol selected, the radiation dose from these external sources can vary from a small fraction to an amount exceeding the injected radiopharmaceutical dose.

Line Sources. In 1983, Carrol defined a method for measuring a system of line sources to obtain a patient-specific attenuation scan and this system is still in use. ${ }^{17}$ This method can provide a reliable attenuation map at a very low patient dose (e.g., $0.04 \mathrm{mSv}$ )..$^{15,18}$ Recent advances in iterative transmission reconstruction algorithms significantly improve the quality of the reconstructed transmission scans using as little as 60 seconds of acquisition times. ${ }^{19}$ See Figure 1. The major limitations of line source systems are that transmission imaging must be acquired in a 2D mode and septa must be retracted before 3D emission imaging. Another limitation is the limited availability of new line source systems, as only one manufacturer produces a new PET only system. Most systems are supported by refurbished market suppliers. Despite the lack of new instrumentation, a large fraction of cardiac PET scanners in use employ line source systems.

\section{X-ray CT-Based Attenuation Correc-} tion. PET/CT systems employ a conventional multislice x-ray CT system capable of acquiring highresolution, diagnostic quality data and/or low-dose attenuation correction scans. ${ }^{20}$ Radiation from CT-based attenuation systems can be as little as $0.3 \mathrm{mSv}^{21}$ to upwards of $8.5 \mathrm{mSv},{ }^{22}$ the latter dose being over twice the radiation dose from a ${ }^{82} \mathrm{Rb}$ perfusion examination. Minimizing the radiation dose means using appropriate protocols for cardiac PET/CT attenuation correction.

Several protocols have been introduced to ensure high-quality transmission scanning. CT scans have better signal-to-noise than line source attenuation scans. However, CT-specific artifacts, such as breathing 

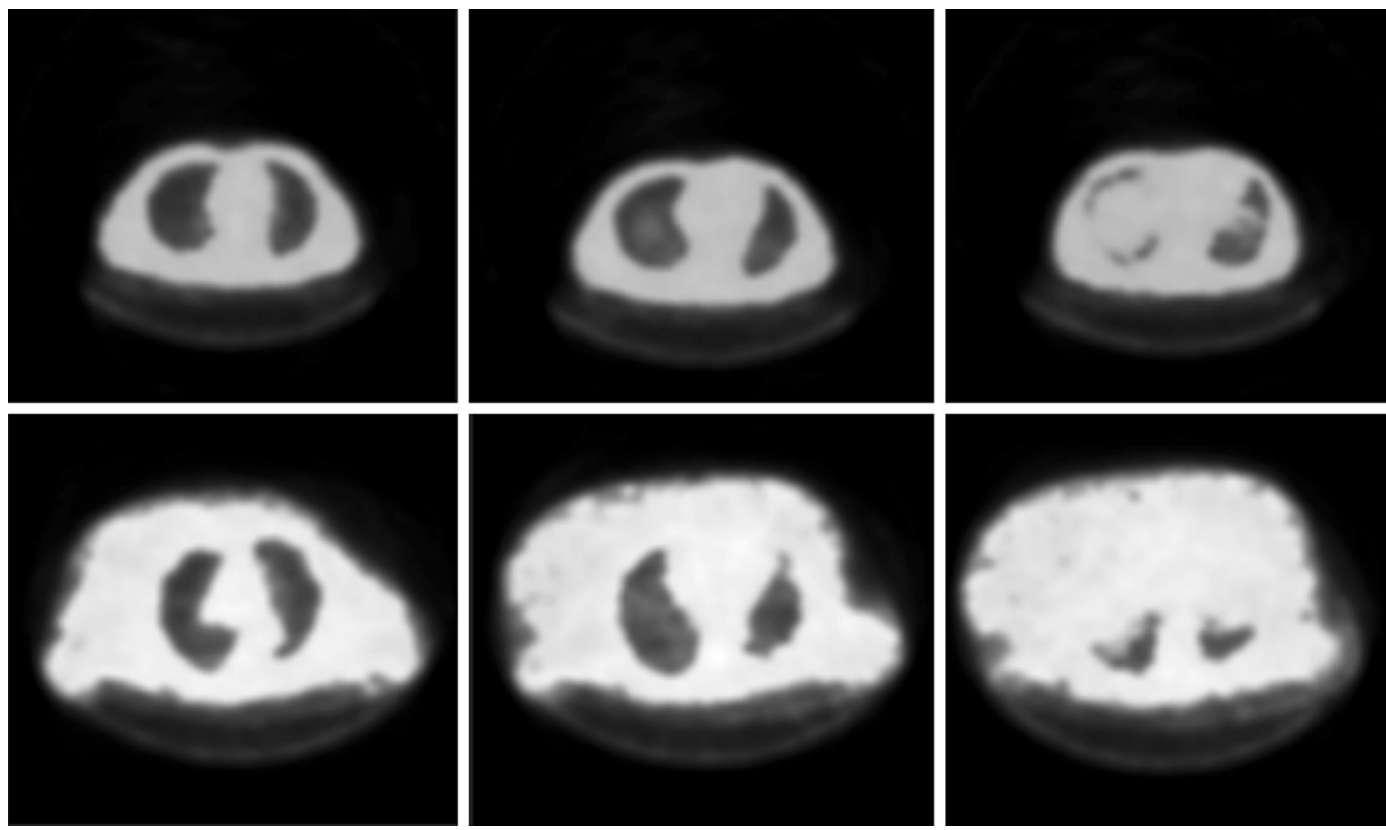

Figure 1. High-quality transmission images acquired using Ge-68 rotating rod sources on a Siemens ACCEL (Siemens Medical Systems, Knoxville TN). These transmission scans can have radiation doses as low as $0.04 \mathrm{mSv}$. The top row is of a normal BMI patient and the bottom row is from a morbidly obese patient. Total scan time was $90 \mathrm{~s}$. Images are shown at three different levels of the heart.

artifacts and metal artifacts associated with pacing and ICD leads, can make PET/CT x-ray-based attenuation correction more challenging than line source attenuation correction. ${ }^{23}$

The CT portion of the cardiac PET/CT may provide additive information to the perfusion study. ${ }^{24}$ Studies that indicate the presence of coronary calcium in patients with no known coronary disease can be helpful in the evaluation of patient risk, and visual analysis of coronary calcium detected with the low-dose CT scan performed for attenuation correction may add useful information without exposing the patient to a full diagnostic CT. ${ }^{25}$

\section{DOSE REDUCTION STRATEGIES}

Cardiovascular PET myocardial perfusion imaging with ${ }^{82} \mathrm{Rb}$, when implemented properly, is a low radiation procedure, with total radiation doses of $4 \mathrm{mSv}$ for typical 2D protocols (50 $\mathrm{mCi}$ for each infusion) and $2 \mathrm{mSv}$ for typical 3D protocols ( $25 \mathrm{mCi}$ for each infusion). ${ }^{11}$ However, achieving these doses requires close adherence to imaging standards and adapting protocols to meet the needs of each patient. Laboratories should be aware that recommended patient doses can vary based on PET instrumentation. ${ }^{25}$ Major reductions can be achieved through (1) the use of 3D imaging, (2) reducing the number of attenuation scans, and (3) use of weightadjusted radioactivity administration.

\section{Improving PET Image Quality While Reducing Radiation with 3D Imaging}

Early PET systems used 2D imaging where a system of lead or tungsten septa is placed between the detector rings to reduce the dead-time and absorb up to $90 \%$ of the scattered photons. Although these 2D imaging systems can produce high-quality images, the septa remove a large proportion of true events that can be used in reconstruction. Lower patient radiation dose and higher count density can be achieved with 3D imaging where the septa are eliminated, thereby increasing the sensitivity of the system by a factor of 2- to 5 times compared with 2D imaging, see Figure $2 .{ }^{26}$

For ${ }^{82} \mathrm{Rb}$ myocardial perfusion studies, ASNC guidelines recommend a $30-40 \mathrm{mCi} \quad(1,110-$ $1,480 \mathrm{MBq}$ ) injected dose for both rest and stress studies when 3D imaging is used, and $40-60 \mathrm{mCi}$ $(1,480-2,220 \mathrm{MBq})$ doses for rest and stress studies with $2 \mathrm{D}$ imaging. ${ }^{15}$ In a study of 70 patients using a weight-adjusted, low-dose 3D-imaging protocol (10 MBq/kg), 3D PET/CT demonstrated excellent sensitivity, specificity, and overall accuracy $(100 \%, 71 \%$, and $89 \%$, respectively), and was comparable to similar 


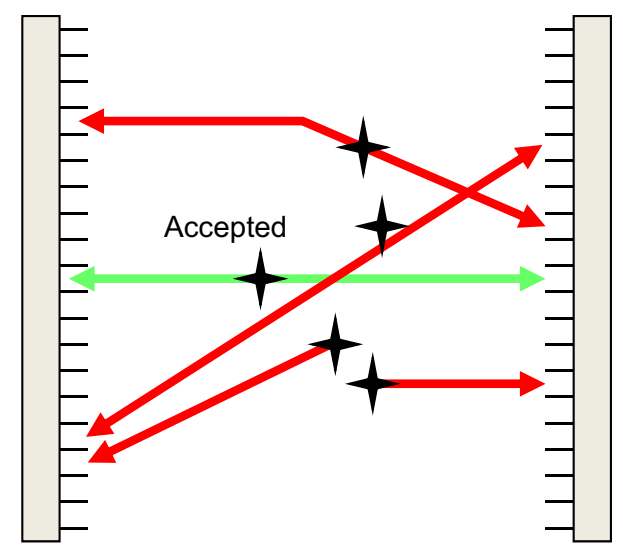

\section{D mode-only parallel photons accepted}

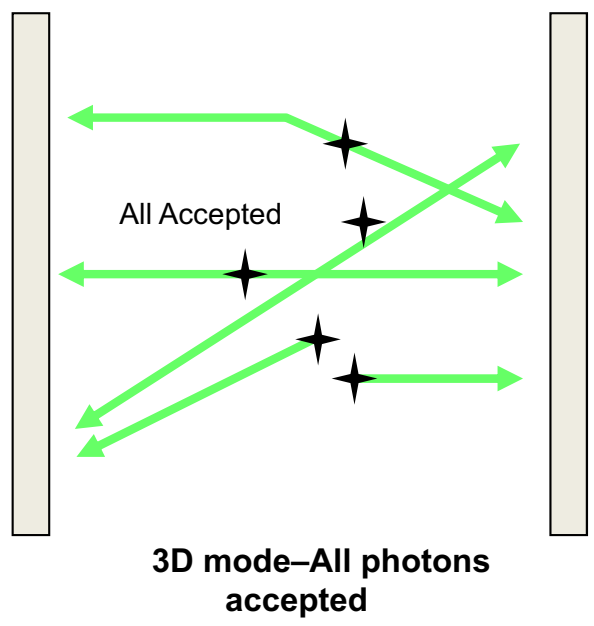

Figure 2. In 2D PET imaging, contamination from noncoincidence and scattered photons is excluded using a system of inter-plane septa. Although these septa are very effective in removing these unwanted photons, it comes at the expense of decreasing the system sensitivity by a factor of $2-5$ compared to 3D PET systems. ${ }^{26}$

studies using 2D PET. $^{27}$ In addition, absolute MBF measurements appear to be equivalent using $2 \mathrm{D}$ and $3 \mathrm{D}$ imaging, if the 3D activity is limited to avoid detector saturation effects. ${ }^{28}$ When available, clinicians should utilize 3D imaging so long as the hardware and software have been validated for the specific cardiac application. Reducing the injected dose from $50 \mathrm{mCi}(1,850 \mathrm{MBq})$ to $25 \mathrm{mCi}(925 \mathrm{MBq})$ results in an average dose reduction of $2 \mathrm{mSv}$ for a typical rest-stress study. Sample imaging protocols for 2D and 3D imaging are given in Figure 3. Current recommendations for injected dose do not differ for ${ }^{13} \mathrm{~N}$-ammonia $(10-20 \mathrm{mCi})$ and ${ }^{18} \mathrm{~F}$ FDG (5-15 mCi) when using $2 \mathrm{D}$ or 3D imaging.
Recommendation: validated 3D imaging should be used whenever possible for both dose reduction and high-quality images.

\section{Optimizing Attenuation Correction Transmission Scan Settings}

A common approach for attenuation correction of PET studies is the use of x-ray CT. The CT image quality required for attenuation correction is much lower than for diagnostic quality images. Specifically, a CT attenuation correction scan is acceptable for attenuation correction if: the $\mathrm{CT}$ attenuation correction scan appears uniform in the soft tissue regions when viewed using a gray scale from $-1,000$ to 1,000 Hounsfield units; the lung boundary is well defined and free of breathing artifact; and the transmission scan is properly registered with the emission data (See Figure 4).

Radiation dose optimization begins using $\mathrm{x}$-ray tube settings $(\mathrm{kVp}, \mathrm{mAs}$, scan length, field of view (FOV), and scan pitch) to achieve the lowest dose. See Table 2. For most applications, these settings can be kept to 100 $\mathrm{kVp}$ and $10 \mathrm{mAs} .{ }^{21}$ The lowest dose CT acquisition mode uses a sequential "prospective ECG-triggered axial scan." This acquisition mode acquires a single slab using a single rotation of the CT scanner followed by moving the table to the next acquisition position. Radiation dose can be kept to $<1 \mathrm{mSv}$ if the $\mathrm{x}$-ray tube is only on for data acquisition needed for attenuation correction. ${ }^{21}$ When prospective triggering is not possible, ECG-based dose modulation is a critical consideration for acquiring transmission data in the retrospective ECG gated helical mode. Most systems include a proprietary dose modulation algorithm and should be used when helical scanning is used. ${ }^{21}$

Using iterative reconstruction of the CT scans can reduce the need to use a high $\mathrm{CT}$ radiation dose to acquire a high-quality CT scan for attenuation correction. ${ }^{29}$ Failure to follow a cardiac specific protocol can result in as much as a tenfold increase in CT radiation.

The choice of a breathing protocol for acquiring the transmission scan has a direct relationship on the radiation dose received by the patient since different scanning parameters are used for each protocol, see Table $3 .^{30-32}$ Three breathing approaches are commonly used to minimize artifacts on the CT images: endexpiration with or without breath holding, shallow freebreathing, and cine-CT. ${ }^{30}$ Because each of these strategies requires different scanning parameters, dose reduction strategies for $\mathrm{CT}$ attenuation correction should be customized to the breathing strategy employed. When 
A

Vasodilator stress

$50 \mathrm{mCi}$

$50 \mathrm{mCi}$

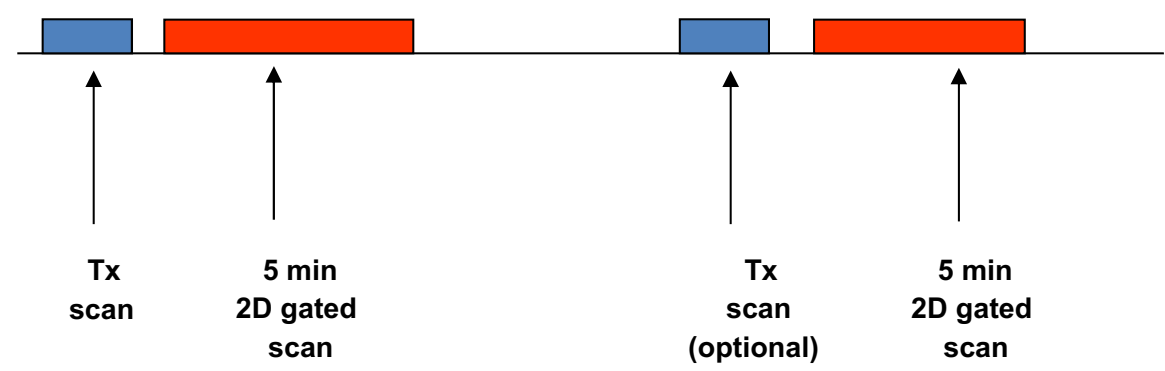

Vasodilator stress

B

$30 \mathrm{mCi} \quad 30 \mathrm{mCi}$

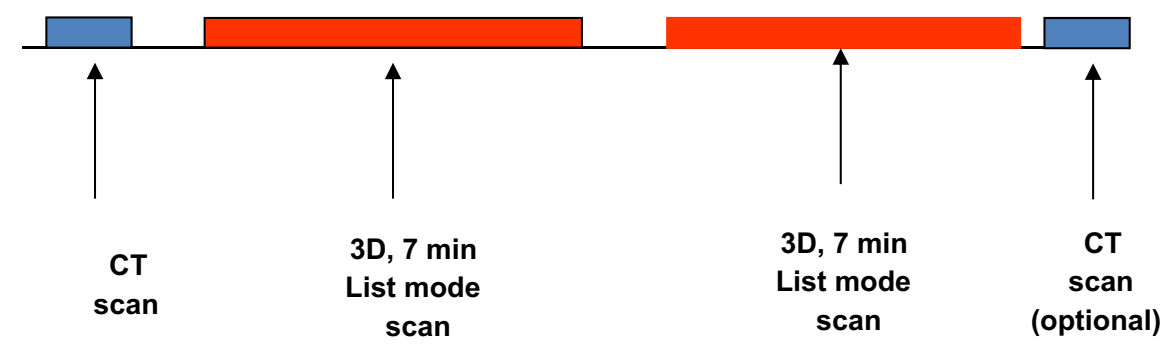

C

Vasodilator stress

$40 \mathrm{mCi}$

$40 \mathrm{mCi}$

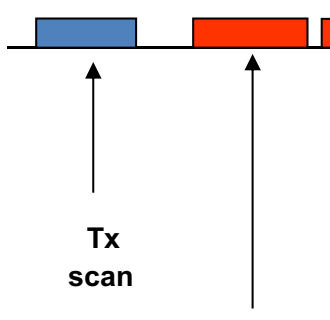

$2.5 \mathrm{~min}$

2D dynamic

scan

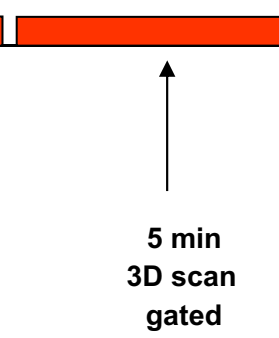

gated

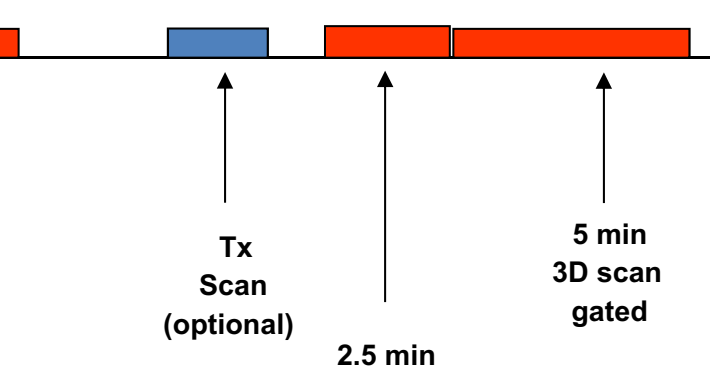

2D dynamic

scan

Figure 3. Dynamic, gated, and ${ }^{82} \mathrm{Rb}$ perfusion data can be acquired in PET using either frame or List-mode. A Standard 2D ECG gated PET protocol, B List-mode dynamic, gated, and perfusion protocol, and C Frame-mode dynamic, gated, and perfusion protocol. It is important when designing an imaging protocol that diagnostic value is not sacrificed for reduced radiation dose.

dose strategies are optimized, the radiation from the CT attenuation correction scan can be as little as $0.14 \mathrm{mSv}$ using end-expiration or free-breathing protocols. $^{33}$
Recommendations: To achieve the lowest CT dose:

- Optimize kVp, mAs, typically $100 \mathrm{kVp}$ or less and $10 \mathrm{mAs}$ or less. 


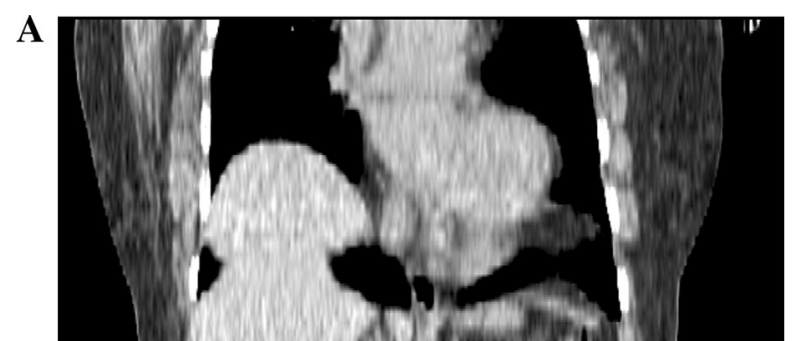

$\mathbf{B}$

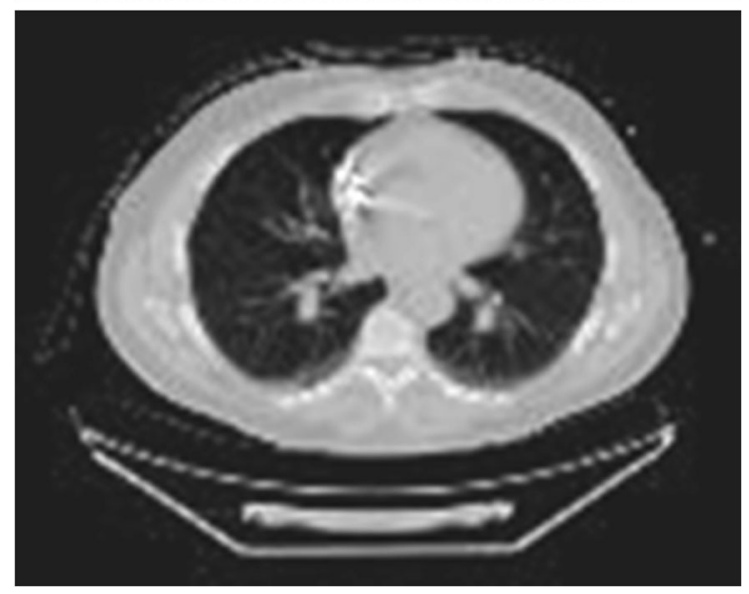

Figure 4. Examples of CT artifacts. A Breathing artifact from a free-breathing protocol. The liver can be seen in two different places. B Metal artifact in a computed tomography attenuation correction image.
- Utilize prospective ECG triggering when possible. If helical scanning is required, dose modulation should be employed.

- Confirm FOV settings for obtaining the attenuation correction scan are confined to the cardiac region only without truncation of the body inplane.

- Avoid cine-CT free-breathing protocols unless the PET/CT system is designed by the manufacturer to perform this scan at a radiation dose comparable to either ECG triggering or helical scanning with dose modulation.

\section{Use of a Single-Transmission Attenuation Scan}

If the patient does not move substantially between the rest and stress perfusion studies, acquiring separate transmission scans may not be necessary. However, if there is extreme patient movement, attenuation correction may not be properly applied without correcting for the patient movement by re-aligning the emission and transmission datasets. ${ }^{23,34}$ Failure to correctly re-align these image sets will result in significant artifact and lower diagnostic accuracy.

Table 2. Relationship between CT protocol settings radiation exposure and image quality

\section{CT setting}

$\mathrm{kVp}$

mAs

Pitch

FOV

\section{Purpose}

Increasing peak high voltage of the $\mathrm{X}$-ray tube, increases energy of the electrons. This increases the mean energy of $\mathrm{x}$-rays

Adjusts the number of electrons being generated by the $x$-ray tube (tube current)

A measure of the amount of overlap between adjacent rotations of a helical CT scan

Plane FOV is the size of the in-plane region scanned in the CT scan

Axial FOV is the size of the axial length of a scan

\section{Effect on radiation} dose

Increasing $\mathrm{kVp}$ increases radiation dose

Increasing $\mathrm{mAs}$ increases radiation dose

Increasing pitch decreases radiation dose

Increasing axial and inplane FOV increases radiation dose

\section{Effect on image quality}

Increasing $\mathrm{kVp}$ reduces contrast, but improves beam penetration

Increasing mAs improves signal-to-noise ratio

Increasing pitch reduces breath hold time

Reducing FOV size to smaller than the size of the chest increases truncation artifacts in CT attenuationcorrected images 
Table 3. Strategies for minimizing the dose from CT attenuation maps and imaging artifacts for the different breathing protocols ${ }^{30-32}$

\begin{tabular}{lll}
\hline CT technique & \multicolumn{1}{c}{ Description } & Techniques for minimizing dose \\
\hline Shallow free breath & $\begin{array}{c}\text { For scanners that require more } \\
\text { than } 10 \text { seconds to cover } \\
\text { heart instruct the patient to } \\
\text { breathe using small breaths } \\
\text { through the study }\end{array}$ & $\begin{array}{l}\text { Reduce current to }<10 \mathrm{mAs} \\
\text { Reduce voltage to }<100 \mathrm{kVp} \text { for lower BMI } \\
\text { patients } \\
\text { Use dose modulation } \\
\text { Use high-pitch acquisition (minimal slice } \\
\text { overlap) }\end{array}$ \\
$\begin{array}{c}\text { End-expiration } \\
\text { breath hold }\end{array}$ & $\begin{array}{l}\text { For scanners that require less } \\
\text { than } 10 \text { seconds to cover } \\
\text { heart instruct the patient to } \\
\text { breathe out slightly then hold } \\
\text { breath }\end{array}$ & $\begin{array}{l}\text { Reduce current to }<10 \mathrm{mAs} \\
\text { Reduce voltage to }<100 \mathrm{kVp} \text { for lower BMI } \\
\text { patients }\end{array}$ \\
$\begin{array}{l}\text { Use dose modulation } \\
\text { Use high-pitch acquisition (minimal slice } \\
\text { overlap) }\end{array}$ \\
Fine-CT & $\begin{array}{c}\text { Proprietary technique. Follow } \\
\text { manufacturers' recommendations }\end{array}$ \\
& cine-CT protocol &
\end{tabular}

Recommendation: Use a single-attenuation correction scan for rest and stress images after verifying or correcting misregistration of the PET images. Technologists should only repeat transmission scanning when uncorrectable motion is present (examples include patient rotation, breathing artifacts, or changes in arm position) or if the original transmission scan has insufficient count density.

\section{Patient Positioning with PET/CT Systems}

Positioning of the heart in the center of the FOV can be challenging with PET because of the small transverse axis FOV of the scanner and the lack of tracer in the patient prior to imaging. Optimal positioning is achieved using a planar CT topogram. ${ }^{35}$

Recommendation: Minimize the region scanned for the topogram to only cover the region of the heart for perfusion imaging.

\section{Patient Positioning with Dedicated PET Systems}

One of the early approaches to positioning with dedicated 2D PET systems was to inject a small amount of ${ }^{82} \mathrm{Rb}$ at rest prior to diagnostic scanning $(5-10 \mathrm{mCi})$. This approach, though effective in locating the heart, exposes the patient to an additional $0.2-0.4 \mathrm{mSv}$ and requires an additional 10 minutes for the generator to return to full strength. With the widespread utilization of iterative reconstruction algorithms for line source transmission scans, the acquisition time of transmission scans for attenuation correction has been reduced to less than 2 minutes. This has allowed more sites to use transmission scans to confirm positioning instead of a small dose of ${ }^{82} \mathrm{Rb}$. This approach introduces no additional radiation exposure and does not impact laboratory efficiency.

Recommendation: For dedicated PET systems, use the transmission scan for positioning instead of a lowdose ${ }^{82} \mathrm{Rb}$ scan.

\section{Use Iterative Reconstruction on All Emission Images}

Iterative reconstruction with attenuation correction should be applied to all cardiac PET studies (perfusion, gated, and dynamic datasets). It can improve transmission scan quality and obviate the need for using higher radiation doses. More recently time-of-flight and resolution recovery reconstruction algorithms have been investigated as techniques for improving image quality. ${ }^{36,37}$ Further study is needed before they can be used routinely for dose reduction. 
Recommendation: Use iterative reconstruction to improve image quality of emission datasets.

\section{Weight-Adjusted Tracer Activities}

Weight-adjusted ${ }^{82} \mathrm{Rb}$ activity administration has been suggested for reducing patient dose. ${ }^{3}$ Although successful in reducing patient dose in SPECT, it is not as easily implemented with PET. Some generator systems in use today require recalibration of the dose delivery system when the requested dose is changed. ${ }^{7}$ Some generator systems do allow for adjusting patient doses without recalibrating the infusion system; however, clinicians should verify this capability with the generator manufacturer before using a weight-adjusted dosing protocol. ${ }^{8,38,39}$

Weight-adjusted dosages for ${ }^{13} \mathrm{~N}$-ammonia are not typically recommended. Although it can lower patient dose, the increased handling and the uncertainty of when a patient is ready for imaging make weight adjustment difficult. Weight-adjusted dosing of ${ }^{18} \mathrm{~F}$ FDG cardiac studies has not been well studied, ${ }^{40}$ but there are reports of success in using weight-adjusted doses in sarcoid imaging. ${ }^{41}$

Recommendation: Weight-adjusted dosing of ${ }^{82} R b$ may reduce patient radiation dose in smaller patients and should be considered when using generator systems that can accommodate rapid and reliable dose changes.

\section{APPLYING DOSE REDUCTION STRATEGIES TO CLINICAL PRACTICE}

The implementation of dose reduction strategies must be made with careful attention to the impact on image quality and the diagnostic accuracy of the test. Optimization should be made in increments starting with those having the greatest impact on reducing dose with the least impact on image quality. Table 4 outlines a practical approach towards radiation reduction.

\section{Select the Correct Patient for PET}

Recently, a joint statement from ASNC and the Society of Nuclear Medicine and Molecular Imaging was published stating that rest-stress myocardial perfusion PET is a first-line preferred test for patients with known or suspected CAD who meet appropriate criteria for a stress-imaging test and are unable to complete a diagnostic-level exercise stress-imaging study. ${ }^{42}$ PET should be considered a preferred test for patients who meet appropriate use criteria for a stress-imaging test and who require pharmacologic stress. ${ }^{42}$

The most recent Appropriate Use Criteria for ischemic heart disease were published in 2014 and should help guide physician decisions regarding the use of cardiovascular myocardial perfusion PET imaging in the most common clinical scenarios. ${ }^{43}$

Table 4. Steps to optimizing radiation dose reduction and maintaining optimal image quality

1. Apply appropriate use criteria for patient selection. Consider the patient's individual risk to radiation

2. Use validated 3D imaging for both dose reduction and consistent high-quality images

3. Consider weight-adjusted dosing of ${ }^{82} \mathrm{Rb}$ when generator systems support patient-specific dosing

4. Optimize radiation dose of the CT scan in PET/CT using $x$-ray tube settings $(k V p, m A s$, scan length, field of view (FOV), and scan pitch) to achieve the lowest dose for attenuation correction

5. Use a single-attenuation map for rest and stress after verifying correct alignment and re-aligning the image sets if there is misregistration

6. Adhere to recommended breathing protocol strategies for attenuation map acquisition

7. Reduce dose for patient positioning

-For dedicated PET systems use the transmission study for positioning instead of an additional ${ }^{82} \mathrm{Rb}$ dose

-For PET/CT system minimize the dose used for the topogram

8. For perfusion studies, extract all information possible from a given dose, including perfusion, gating, and flow data

9. Use iterative reconstruction methods for all emission datasets

10. When accessing radiation risk, consider all sources of radiation including emission injection, transmission study, and any comparators tests needed to perform the assessment

11. Radiation protection strategies must also include protection for the staff and public, including appropriate shielding for $511 \mathrm{keV}$ photons and CT systems 


\section{Utilize 3D Imaging Whenever Possible}

When validated software and hardware are available, 3D PET imaging protocols can result in $\approx 50 \%$ reduction in patient dose compared with $2 \mathrm{D}$ imaging. Because of the complexity of the 3D reconstruction algorithms, with increased scatter and dead-time of the scanner in 3D mode, facilities must carefully inspect the quality of output images to verify image quality is not sacrificed when switching from 2D to 3D imaging. When using 3D PET for ${ }^{82} \mathrm{Rb}$ perfusion imaging, the reconstruction algorithm should include correction for the added prompt gamma background that is not present with other cardiac PET tracers.

\section{Obtain Low-Dose Radiation Attenuation- Correction Scans}

For most cardiac PET studies, diagnostic quality $\mathrm{x}$ ray CT data are unnecessary and should not be performed. Minimizing $\mathrm{mAs}$ and $\mathrm{kVp}$ for attenuation correction scans can reduce patient dose. In large BMI patients, facilities should be prepared to increase these settings to obtain usable CT data for attenuation correction.

\section{Avoid Repeated Attenuation Scanning}

For sequential rest-stress myocardial perfusion studies, facilities should consider obtaining a second, post-stress transmission scan only when there is evidence of patient motion. Technologists should carefully inspect the quality of the rest transmission scan for registration and image quality. If resting transmission scan data are appropriately registered with the stress emission data, repeating the transmission scan for stress is not necessary.

Facilities should avoid using a non-diagnostic lowdose radiotracer scan for positioning and instead rely on transmission scan data to confirm patient positioning prior to testing.

It is important to perform the minimum number of image sets in the assessment of myocardial viability. The rest and stress or rest-only images should be reviewed and if there is a large area of ischemia on rest and stress or a normal resting perfusion study, the ${ }^{18} \mathrm{~F}$ FDG images do not add additional information and should be canceled.

\section{Extract All Information Possible from a Given Dose}

Facilities should acquire data in such a way as to extract maximal information from the injected dose: relative perfusion, absolute $\mathrm{MBF}$, flow reserve, wall motion, and LVEF to avoid the need for additional testing and increased exposure. In addition, visual assessment of coronary calcium can provide additive information even when a diagnostic CT study is not acquired.

\section{Use Iterative Reconstruction Methods}

Iterative reconstruction with attenuation correction should be applied on all cardiac PET studies. When available, iterative reconstruction of CT and line sourcebased attenuation can allow for lower dose transmission studies. When advanced image reconstruction algorithms are available and validated, they may allow for administration of smaller doses.

\section{Consider All Sources of Radiation}

The injected tracer activity is not the only source of radiation. When determining the radiation dose, it is important to include the transmission scan and any comparator test needed to interpret the clinical data. Facilities should avoid repeating comparator tests.

\section{Consider Radiation Exposure to the Staff and the Public}

Imagers not only have a responsibility to reduce radiation dose to the patient, but they also must consider radiation dose to the staff and public. Adequate shielding must be present and appropriate for the high-energy $511 \mathrm{keV}$ photons and the CT x-rays in hybrid systems. Placement of the imaging suite should be a safe distance from any waiting areas. Adequate lead shielding must include the CT system. When using ${ }^{8} \mathrm{~F}$ FDG and ${ }^{13} \mathrm{~N}$ ammonia, the technologist must use an appropriate syringe shield.

\section{CONCLUSION}

Appropriate use of cardiovascular PET imaging provides the opportunity to obtain highly accurate and often unique clinical information at potentially very low risk of radiation exposure. Low radiation exposure is an intrinsic advantage of cardiovascular PET radiopharmaceuticals and instrumentation. Optimizing these advantages requires careful patient-specific attention to selection, acquisition, and processing protocols. However, lowering exposure must never compromise image quality and diagnostic accuracy. Adherence to the recommended dose reduction strategies outlined will give the highest quality diagnostic images with the lowest achievable radiation exposure. 


\section{Acknowledgement}

The authors wish to thank Drs. Michael Desiderio, Matthew Parker, and Raymond Russell for their independent review and insightful comments on this manuscript.

\section{Disclosures}

$R$. deKemp is a consultant for, and receives unrestricted grant funding and rubidium PET technology license revenues from Jubilant DraxImage Inc. $R$. deKemp receives software license revenues from INVIA Medical Imaging Solutions. J. Case is an owner of Cardiovascular Imaging Technologies, L.L.C., that produces PET processing software. P. Slomka participates in software royalties at Cedars-Sinai Medical Center for licensing of nuclear cardiology software and has received research grant support from Siemens Medical Systems. M. Smith receives research support from Siemens Medical Solutions USA, Inc. G. Heller is a Medical Advisor for Molecular Imaging Services and is a consultant to Jubliant DraxImage Inc. M. Cerqueira is a consultant to Astellas Pharma, USA and member of the Astellas Speakers Bureau.

\section{APPENDIX 1: USEFUL DEFINITIONS OF RADIATION}

\section{Activity (A)}

The number of disintegrations per second (dps) and the amount of administered radioactivity is usually given in units of megabecquerels $(\mathrm{MBq})$ or millicuries $(\mathrm{mCi})$. One $\mathrm{Bq}$ is equal to one decay per second and one millicurie is 37 million decays per second.

\section{Absorbed Dose (D)}

The energy per unit mass deposited in tissue is defined to be the absorbed dose, $D$, and is measured in units of gray (Gy) or radiation absorbed dose (rad). One Gy is equal to one joule/ $\mathrm{kg}$ and equals $100 \mathrm{rad}$. This unit is independent of the biological impact the radiation has on tissue.

\section{Equivalent Dose $\left(\mathrm{H}_{\mathrm{T}}\right)$}

The equivalent dose takes into consideration the relative biological damage to tissue caused by radiation. It is computed by multiplying the absorbed dose by a weighting factor $W_{\mathrm{R}}$ that is dependent on the radiation type and particle energy, so that $H_{\mathrm{T}}=D \times W_{\mathrm{R}}$. For $\mathrm{PET}$ and PET/CT radiation, the weighting factor is one for emitted positrons and photons. The units of equivalent dose are sievert (Sv) or roentgen equivalent man (rem). An absorbed dose in Gy or rad times a weighting factor of one yields the same numerical value in $\mathrm{Sv}$ or rem.

\section{Whole Body Dose}

The whole (or total) body dose is defined as the sum of the energy deposited in each organ or body tissue, with applied radiation weighting factors $W_{R}$, divided by the body mass. This is of limited value in nuclear medicine due to non-uniform tracer distribution and has been replaced by measures that reflect the radiation sensitivity of different organs.

\section{Effective Dose Equivalent $\left(\mathrm{H}_{\mathrm{E}}\right)$}

The effective dose equivalent, $H_{\mathrm{E}}$, was a weighted average of the equivalent dose using tissue or organ weights, $W_{\mathrm{T}}$, given by the International Commission on Radiological Protection (ICRP) in 1979 [ICRP 1979]. The sum of the tissue weights is one. The ICRP has updated this concept with the effective dose, $E$, with new weighting factors (see below).

\section{Effective Dose (E)}

The effective dose is a tissue weighted average of the equivalent dose in each individual organ. Specifically, effective dose $(E)$ can be expressed as

$$
E=\sum_{T} W_{T} H_{T}
$$

where $H_{\mathrm{T}}$ is the equivalent dose absorbed by the Tth organ and $W_{\mathrm{T}}$ is the weighting factor for that organ, reflecting the estimated cancer risk. The sum of the weights is one. Adult organ and total body dosimetry values are usually based on simplified kinetic models and an idealized $70-\mathrm{kg}$ person.

\section{References}

1. International Atomic Energy Agency. Radiological protection for medical exposition to ionizing radiation. IAEA Safety Standard, 2002:RS-G-15.

2. US Food and Drug Administration. Initiative to reduce unnecessary radiation exposure from medical imaging, , February 2010, http://www.fda.gov/downloads/Radiation-EmittingProducts/Radia tionSafety/RadiationDoseReduction/UCM200087.pdf

3. Cerqueira MD, Allman KC, Ficaro EP, Hansen CL, Nichols KJ, Thompson RC, et al. American Society for Nuclear Cardiology Information Statement: Recommendations for reducing radiation exposure in myocardial perfusion imaging. $\mathrm{J}$ Nucl Cardiol. 2010;17:709-18.

4. Piccinelli M, Garcia EV. Advances in software for faster procedure and lower radiotracer dose myocardial perfusion imaging. Prog Cardiovasc Dis. 2015;57:579-87. 
5. Hill KD, Einstein AJ. New approaches to radiation exposure. Trends Cardiovasc Med. 2016;26:55-65.

6. Einstein AJ, Blankstein R, Andrews H, Fish M, Padgett R, Hayes $\mathrm{SW}$, et al. Comparison of image quality, myocardial perfusion, and left ventricular function between standard imaging and single-injection ultra-low-dose imaging using a high-efficiency SPECT camera: the MILLISIEVERT study. J Nucl Med. 2014;55:1430-7.

7. Bracco Diagnostics. [Prescribing Information] CardioGen- $82^{\circledR}$ (Rubidium Rb 82 Generator), Bracco Diagnostics (NJ, USA) revised April 2013. Available at: http://imaging.bracco.com/sites/ braccoimaging.com/files/technica_sheet_pdf/Cardiogen $\% 20$ Full\% 20Prescribing\%20Information.pdf. Accessed June 30, 2016.

8. Jubilant DRAXIMAGE. [Prescribing Information] Ruby-Fill ${ }^{\circledR}$ (rubidium $\mathrm{Rb} 82$ generator), Jubilant DRAXIMAGE (Kirkland Quebec). Available at: http://www.draximage.com/DATA/TEX TEDOC/Approval-202153-Ruby-Fill-Labeling-30Sept-2016.pdf. Accessed October 1, 2016.

9. Senthamizhchelvan S, Bravo PE, Esaias C, Lodge MA, Merrill J, Hobbs RF, et al. Human biodistribution and radiation dosimetry of 82Rb. J Nucl Med. 2010;51:1592-9.

10. International Commission on Radiological Protection, 2015. Radiation dose to patients from radiopharmaceuticals: A compendium of current information related to frequently used substances. ICRP Publication 128. Ann. ICRP 44(2S).

11. Hunter CR, Hill J, Ziadi MC, Beanlands RS, deKemp RA. Biodistribution and radiation dosimetry of $(82) \mathrm{Rb}$ at rest and during peak pharmacological stress in patients referred for myocardial perfusion imaging. Eur J Nucl Med Mol Imaging. 2015;42:1032-42.

12. Saha GB. Fundamentals of nuclear pharmacy. 5th ed. New York: Springer; 2004.

13. Schindler TH, Schelbert HR. Quantitation of myocardial perfusion: absolute blood flow versus relative uptake. In: Dilsizian V, Narula J, editors. Atlas of nuclear cardiology. 4th ed. New York: Springer; 2013.

14. Taegtmeyer H, Dilsizian V. Imaging cardiac metabolism. In: Dilsizian V, Narula J, editors. Atlas of nuclear cardiology. 4th ed. New York: Springer; 2013.

15. Dilsizian V, Bacharach SL, Beanlands SR, Bergmann SR, Delbeke $\mathrm{D}$, Dorbala $\mathrm{S}$, et al. ASNC imaging guidelines/SNMMI procedure standard for positron emission tomography (PET) nuclear cardiology procedures. J Nucl Cardiol. 2016;23:1187-226.

16. Dorbala S. Standard myocardial perfusion and cardiac FDG PET protocols and associated patient radiation doses. http://www.ima gewisely.org/ /media/ImageWisely\%20Files/NucMed/Standard\% 20Myocardial\%20Perfusion.pdf. Accessed, May 10, 2016.

17. Carroll LR, Kertz P, Orcut O. The orbiting rod source: improving performance in PET transmission correction scans. In: Esser PD. Emission computed tomography: Current trends. New York: The Society of Nuclear Medicine; 1983, p 235-247.

18. Slomka PJ, Pan T, Germano G. Recent advances and future progress in PET instrumentation. Semin Nucl Med. 2016;46:5-19.

19. Hsu BL, Case JA, Moser KW, Bateman TM, Cullom SJ. Reconstruction of rapidly acquired germanium- 68 transmission scans for cardiac PET attenuation correction. J Nucl Cardiol. 2007;14:706-14.

20. Kinahan PE, Townsend DW, Beyer T, Sashin D. Attenuation correction for a combined 3D PET/CT scanner. Med Phys. 1998;25:2046-53.

21. Dorbala S, Di Carli MF, Delbeke D, Abbara S, DePuey EG, Dilsizian V, et al. SNMMI/ASNC/SCCT guideline for cardiac SPECT/CT and PET/CT 1.0. J Nucl Med. 2013;54:1485-507.

22. Wu TH, Huang YH, Lee JJ, Wang SY, Wang SC, Su CT, et al. Radiation exposure during transmission measurements:
Comparison between CT- and germanium-based techniques with a current PET scanner. Eur J Nucl Med Mol Imaging. 2004;31:38-43.

23. Gould L, Pan T-S, Laghin C, Johnson NP, Guha A, Sdringola S. Frequent diagnostic errors in cardiac PET/CT due to misregistration of CT attenuation and emission PET images: A definite analysis of causes, consequences and corrections. J Nucl Med. 2007;48:1112-21.

24. Chang S, Nabi F, Xu J, Peterson LE, Achari A, Pratt CM, et al. The coronary artery calcium score and stress myocardial perfusion imaging provide independent and complementary prediction of cardiac risk. J Am Coll Cardiol. 2009;54:1872-82.

25. Einstein AJ, Johnson LL, Bokhari S, Son J, Thompson RC, Bateman TM, et al. Agreement of visual estimation of coronary artery calcium from low-dose CT attenuation correction scans in hybrid PET/CT and SPECT/CT with standard Agatston Score. J Am Coll Cardiol. 2010;56:1914-21.

26. Cherry SR, Dahlborn M, Hoffman EJ. 3D PET using a conventional multislice tomography without septa. J Comput Assist Tomogr. 1991;15:655-68.

27. Knesaurek K, Machac J, Krynyckyi BR, Almeida OD. Comparison of 2-dimensional and 3-dimensional $82 \mathrm{Rb}$ myocardial perfusion PET imaging. J Nucl Med. 2003;4:1350-6.

28. Lekx KS, deKemp RA, Beanlands RS, Wisenberg G, Wells G, Stodilka RZ, et al. 3D versus $2 \mathrm{D}$ dynamic ${ }^{82} \mathrm{Rb}$ myocardial blood flow imaging in a canine model of stunned and infarcted myocardium. Nucl Med Commun. 2010;31:75-81.

29. Padole A, Ali Khawaja RD, Kalra M, Singh S. CT radiation dose and iterative reconstruction techniques. AJR Am J Roentgenol. 2015;204:W384-92.

30. de Juan R, Seifert B, Berthold T, von Schulthess GK, Goerres GW. Clinical evaluation of a breathing protocol for PET/CT. Eur Radiol. 2004;14:1118-23.

31. Alessio AM, Kohlmyer S, Branch K, Chen G, Caldwell J, Kinahan P. Cine CT for attenuation correction in cardiac PET/CT. J Nucl Med. 2007;48:794-801.

32. Goerres GW, Kamel E, Heidelberg TN, Schwitter MR, Burger C, von Schulthess GK. PET-CT image co-registration in the thorax: Influence of respiration. Eur J Nucl Med Mol Imaging. 2002;29:351-60.

33. Xia T, Alessio AM, De Man B, Manjeshwar R, Asma E, Kinahan PE. Ultra-low dose CT attenuation correction for PET/CT. Phys Med Biol. 2012;57:309-28.

34. Case JA, Bateman TM. Taking the perfect nuclear image: Quality control, acquisition, and processing techniques for cardiac SPECT, PET, and hybrid imaging. J Nucl Cardiol. 2013;20:891-907.

35. O'Daniel JC, Stevens DM, Cody DD. Reducing radiation exposure from survey CT scans. AJR Am J Roentgenol. 2005;185:509-15.

36. Akamatsu G, Ishikawa K, Mitsumoto K, Taniguchi T, Ohya N, Baba S, et al. Improvement in PET/CT image quality with a combination of point-spread function and time-of-flight in relation to reconstruction parameters. J Nucl Med. 2012;53:1716-22.

37. Teoh EJ, McGowan DR, Macpherson RE, Bradley KM, Gleeson FV. Phantom and clinical evaluation of the Bayesian penalized likelihood reconstruction algorithm Q. Clear on an LYSO PET/CT system. J Nucl Med. 2015;56:1447-52.

38. Kaster T, Mylonas I, Renaud JM, Wells GA, Beanlands RS, deKemp RA. Accuracy of low dose rubidium-82 myocardial perfusion imaging for detection of coronary artery disease using 3D PET and normal database interpretation. J Nucl Cardiol. 2012;19:1135-45.

39. Klein R, Adler A, Beanlands RS, de Kemp RA. Precision-controlled elution of a $82 \mathrm{Sr} / 82 \mathrm{Rb}$ generator for cardiac perfusion imaging with positron emission tomography. Phys Med Biol. 2007;52:659-73. 
40. Lindner O, Pascual TN, Mercuri M, Acampa W, Burchert W, Flotats A, et al. Nuclear cardiology practice and associated radiation doses in Europe: results of the IAEA Nuclear Cardiology Protocols Study (INCAPS) for the 27 European countries. Eur J Nucl Med Mol Imaging. 2016;43:718-28.

41. Skali H, Schulman AR, Dorbala S. 18F-FDG PET/CT for the assessment of myocardial sarcoidosis. Curr Cardiol Rep. 2013; 15:352.

42. Bateman TM, Dilsizian V, Beanlands RS, DePuey EG, Heller GV, Wolinsky DA. American Society of Nuclear Cardiology and Society of Nuclear Medicine and Molecular Imaging Joint Position Statement on the Clinical Indications for Myocardial Perfusion PET. J Nucl Cardiol. 2016;23:1227-31.
43. Ronan G, Wolk MJ, Bailey SR, Doherty JU, Douglas PS, Hendel RC, et al. ACCF/AHA/ASE/ASNC/HFSA/HRS/SCAI/SCCT/ SCMR/STS 2013 multimodality appropriate use criteria for the detection and risk assessment of stable ischemic heart disease: a report of the American College of Cardiology Foundation Appropriate Use Criteria Task Force, American Heart Association, American Society of Echocardiography, American Society of Nuclear Cardiology, Heart Failure Society of America, Heart Rhythm Society, Society for Cardiovascular Angiography and Interventions, Society of Cardiovascular Computed Tomography, Society for Cardiovascular Magnetic Resonance, and Society of Thoracic Surgeons. J Nucl Cardiol. 2014;21:192-220. 